\title{
Review of: "Predicting Poststroke Pneumonia in Patients With Anterior Large Vessel Occlusion: A Prospective, Population-Based Stroke Registry Analysis"
}

\author{
Fabrizio Giammello ${ }^{1}$ \\ 1 University of Messina \\ Potential competing interests: The author(s) declared that no potential competing interests exist.
}

This is a prospective observational analysis based on stroke registry covering the 119 hospitals within the entire federal state of Hesse, Germany. They included data for 4,281 patients with acute ischemic stroke (AIS) due to large vessel occlusion (LVO) of the anterior circulation, with the aim of assessing predictive factors for poststroke pneumonia (PSP). From all patients with LVO, 19.4\% $(n=832)$ developed PSP, defined by using a combination of imaging, clinical, and laboratory criteria in accordance with guidelines. The study compared three different scores for the prediction of PSP (A2DS2, PNEUMONIA, and ISAN), in order to determine their validity. The principal finding was that among LVO patients this risk of PSP was further increased if an endovascular treatment (EVT) was performed, and if patients underwent intensive care unit (ICU) treatment. Other predictive factors - such as severity of the stroke syndrome and impaired consciousness at admission, male sex, age above 65 years, dysphagia, as well as the comorbidities atrial fibrillation and diabetes - confirmed that LVO patients are at an overall higher risk of developing PSP, accordingly with previous findings. Despite significant limitations due to insufficient information in their registry, they claim that multivariate analysis identified EVT (OR 1.5) and combined EVT plus IVT (OR 1.5) as significant independent risk factors for PSP.

This is a valuable work over predictive factors for PSP risk, especially considering the assessment of predictive validity for PSP scores that may be easily applied in the stroke setting. The paper is well-written, the methods are clear, and the conclusions properly derived from the results.

I have only one concern with this study. The authors stated that neurointerventionalists were free to perform EVT under conscious sedation (CS) or general anesthesia (GA) and to choose the preferred method for EVT individually within the study centers. Nevertheless, the type of anesthesia was not recorded in the registry, and therefore no data was available on whether patients received GA or CS during EVT in the hospitals. As the authors claimed in limitations paragraph, they assumed that "a vast majority of patients receiving EVT were treated under GA because it is considered the standard procedure in Hesse, but no data on the modality was recorded". Current guidelines cannot provide recommendations to use GA or CS in patients undergoing EVT, even though experts suggest that CS may be favored over GA ${ }^{1-3}$. Actually, EVT has been associated with lower rates of functional independence, higher incidence of PSP, and periprocedural hypotension under GA than under $\mathrm{CS}^{4}$. Furthermore, the possible need for prolonged invasive ventilation and weaning from the respirator may be a risk factor for PSP ${ }^{5}$. However, although several retrospective studies suggest that GA produces worsening of functional outcomes, to date RCTs comparing GA with CS failed to show superiority of either treatment for the primary clinical endpoint 6,7 . The lack of subgroup analysis for the type of anesthesia may be a major 
issue of the population-based registry study, because it might be crucial to relate the risk of PSP to GA rather than to EVT itself.

In addition, the method of EVT was not recorded in the registry either, and therefore further questions may be asked over how the use of different endovascular techniques could affect the manifestation of PSP. Hence, it might be even more important to distinguish between the types of anesthesia, in order to establish a correlation of PSP to GA rather than endovascular techniques.

\section{References}

1. Turc, G. et al. European Stroke Organisation (ESO) - European Society for Minimally Invasive Neurological Therapy (ESMINT) Guidelines on Mechanical Thrombectomy in Acute Ischaemic StrokeEndorsed by Stroke Alliance for Europe (SAFE). Eur. Stroke J.4, 6-12 (2019).

2. Ahmed, N. et al. Consensus statements and recommendations from the ESO-Karolinska Stroke Update Conference, Stockholm 11-13 November 2018. Eur. Stroke J. 4, 307-317 (2019).

3. Powers, W. J. et al. 2018 Guidelines for the Early Management of Patients With Acute Ischemic Stroke: A Guideline for Healthcare Professionals From the American Heart Association/American Stroke Association. Stroke 49, e46-e110 (2018).

4. Eker, O. F. et al. Impact of anesthetic management on safety and outcomes following mechanical thrombectomy for ischemic stroke in SWIFT PRIME cohort. Front. Neurol. 9, 1-9 (2018).

5. Zhu, Y., Gao, J., Lv, Q., Yin, Q. \& Yang, D. Risk Factors and Outcomes of Stroke-Associated Pneumonia in Patients with Stroke and Acute Large Artery Occlusion Treated with Endovascular Thrombectomy. J. Stroke Cerebrovasc. Dis. 29, 105223 (2020).

6. Hendén, P. L. et al. General Anesthesia Versus Conscious Sedation for Endovascular Treatment of Acute Ischemic Stroke: The AnStroke Trial (Anesthesia during Stroke). Stroke 48, 1601-1607 (2017).

7. Schönenberger, S. et al. Effect of conscious sedation vs general anesthesia on early neurological improvement among patients with ischemic stroke undergoing endovascular thrombectomy: A randomized clinical trial. JAMA - J. Am. Med. Assoc. 316, 1986-1996 (2016). 